\title{
Briefly Legal: Failure to Safely Deliver Timely and A Botched Resuscitation Lead to Adverse Outcome
}

Maureen E. Sims, M.D., Barry Schifrin, M.D,

\section{Case Presentation}

The patient was a 29-year-old primigravida presented at 40 weeks' gestation who presented for elective induction of labor. Her prenatal course had been uncomplicated. External monitoring was done and initially showed a Category I pattern. Artificial rupture of membranes (AROM) produced clear fluid 14 hours prior to delivery. After several more hours of labor, excessive uterine activity was noted, followed by multiple deep variable decelerations with the eventual rise in baseline and loss of variability (a Cat II tracing). When active pushing began, late decelerations appeared with a nadir to the 70s. Pitocin was discontinued intermittently. Despite the evolution of the FHR pattern, the obstetrician decided to allow the patient to pursue operative vaginal delivery- assisted by vacuum.

\section{"When active pushing began, late decelerations appeared with a nadir to the 70s. Pitocin was discontinued intermittently. Despite the evolution of the FHR pattern, the obstetrician decided to allow the patient to pursue operative vaginal delivery- assisted by vacuum."}

The duration of the 2 nd stage of labor was $2 \mathrm{~h} 28 \mathrm{~min}$. Some descent was noted from a $2+$ to a $3+$ station with the vacuum. However, after 17 minutes, two separate pulls were made with the vacuum, decelerations were noted, and delivery by vacuum was abandoned. A knee-chest position with resuscitative efforts was initiated. Eventually, a decision was made to perform a stat cesarean section. While the operating room was being prepared, the fetus crowned. The vacuum was reapplied, which resulted in the delivery of the head, but shoulder dystocia was immediately encountered. With assistance, the patient was placed into McRoberts position, and suprapubic pressure was applied to disengage the right anterior shoulder from behind the pubic symphysis. When that initiative failed, a Woods maneuver was performed during which a (terminal) fetal bradycardia ensued. The shoulder dystocia lasted about 2 minutes. At delivery, the lifeless baby had a tight nuchal cord. The baby was placed on the mother's chest. Cord arterial pH was 6.76 , pC02 was $107 \mathrm{mmHg}$, p02 was 8.7 and base excess was -28.2 (lactate $18.7 \mathrm{mmol} / \mathrm{L}$ ); cord venous $\mathrm{pH}$ was 7.09 , pC02 was $49 \mathrm{mmHg}$, p02 was 51 and base excess was -17.3 (lactate $12.67 \mathrm{mmol} / \mathrm{L}$ ). After 22 minutes of resuscitation, a heart rate (HR) was detected.

A labor and delivery nurse began positive pressure ventilation (PPV) via mask and called a code blue. Ten minutes later, a nurse anesthetist arrived and intubated the infant with a 3.0 ETT to a depth of $10 \mathrm{~cm}$ and gave $0.4 \mathrm{mg}$ epinephrine via the ETT. PPV continued via the ETT. At 15 minutes, the emergency room physician arrived and placed a 20 gauge angiocatheter directly into an umbilical vessel and gave a bolus of NS followed by 1 $\mathrm{mg}$ of atropine, 2 meq of bicarbonate, and two additional doses of epinephrine. On four occasions, the nurses failed to place a peripheral intravenous (PIV) line. At 27 minutes, an arterial gas drawn from the right radial artery had a $\mathrm{pH}$ of $<6.89$, a PC02 of 91 $\mathrm{mmHg}$, a p02 of $123 \mathrm{mmHg}$, and a base excess of-17.9 (lactate $>20$ $\mathrm{mmol} / \mathrm{L})$. At an hour of life, the baby's arterial blood gas showed a pH of 7.18, a pC02 of 20.8, a p02 of 46, and a base excess of18.8 (lac $>20 \mathrm{mmol} / \mathrm{L}$ ). The point of care glucose was $<40 \mathrm{mgdL}$ (a more specific value was not documented). The HR was 163 beats per minute (bpm), and the blood pressure (BP) was 79/54 with a mean of $58 \mathrm{mmHg}$. Fifty percent dextrose was ordered by the emergency room physician to be administered through the catheter still inserted into an umbilical vessel at a rate of $5 \mathrm{ml} / \mathrm{h}$. Given the considerable forewarning, the plaintiff neonatologist was critical of the lack of a skilled resuscitation team present before or immediately after birth, the lack of timely intubation, wrong size endotracheal tube (ETT), wrong insertion depth, lack of placement of an umbilical venous catheter (UVC), lack of epinephrine administration via the UVC, and lack of appropriate documentation of the resuscitation. Placement of an angiocatheter into an (unidentified) umbilical vessel was reckless, as well as the administration of $50 \%$ dextrose.

One and a half hours after delivery, the transport team arrived and noted that the baby was unresponsive, had no spontaneous movements, had generalized hypotonia and a prominent swelling in the occiput. The physician placed an umbilical venous catheter (UVC) but could not successfully place an umbilical arterial catheter (UAC). The venous gas had a pH of 7.19, a PC02 of 21.9 $\mathrm{mmHg}$, a p02 of $55 \mathrm{mmHg}$, and a base excess of -18.8 . The infant was on the ventilator with moderate pressures and $30 \%$ inspired oxygen. Passive cooling was done, and the baby was transported, during which the BP was $52 / 19$ with a mean of $25 \mathrm{mmHg}$.

"At the receiving hospital, the baby received therapeutic hypothermia (TH) because of his hypoxic-ischemic encephalopathy. His course was complicated by disseminated intravascular coagulopathy (D/C) and seizures. The magnetic resonance imaging (MRI) at eight days showed cortical and deep gray matter necrosis, restricted diffusion in the splenium of the corpus callosum."

At the receiving hospital, the baby received therapeutic hypothermia (TH) because of his hypoxic-ischemic encephalopathy. His course was complicated by disseminated intravascular coagulopathy (DIC) and seizures. The magnetic resonance imaging (MRI) at eight days showed cortical and deep gray matter necrosis, restricted diffusion in the splenium of the corpus callosum. There were also supra and infratentorial subdural hemorrhages 
and parieto-occipital cephalohematoma. She was discharged at two months. Blood cultures were negative. On follow-up, she is developmentally delayed, has cerebral palsy, is cognitively impaired, and has seizures.

The obstetrician and hospital were sued and settled.

\section{Plaintiff allegations against obstetrician:}

1. Failure to properly evaluate and safeguard the well-being of the fetus.

2. Failure to timely appreciate the diminished feasibility of safe vaginal delivery

3. Failure to use proper technique during vacuum-assisted delivery

4. Failure to do a cesarean section timely

5. Failure to anticipate shoulder dystocia and obtain assistance

\section{Defense response:}

1. Standard of care for delivery was met.

2. Should dystocia was not predicable

\section{Plaintiff allegations against the hospital}

1. Failure to have a policy on attendance for high-risk deliveries

2. Failure to have a skilled resuscitation team present before delivery

category II tracings and vacuum assist required the resuscitation team presence

3. Failure to have individuals with knowledge and skills to resuscitate a newborn

intubation was not timely, ETT size and depth were incorrect

the umbilical venous line was not placed, and epinephrine was not administered

placement of angiocatheter into the umbilical vessel was dangerous

administration of $50 \%$ dextrose was dangerous

4. The hospital should not be delivering babies if they are not capable of safeguarding the fetus before and immediately after delivery

Defense response:

It is a small rural hospital and cannot be expected to have personnel and equipment; We do the best we can under the circumstances

"Prevention of fetal asphyxia is preferable to managing the newborn who has suffered a hypoxic-ischemic insult during birth. Modern obstetrics aims to determine the feasibility of safe vaginal delivery, recognize, from an evaluation of the FHR pattern, a compromised fetus before irreversible organ damage occurs, and moderate any deterioration."

\section{Discussion:}

Prevention of fetal asphyxia is preferable to managing the newborn who has suffered a hypoxic-ischemic insult during birth. Modern obstetrics aims to determine the feasibility of safe vaginal delivery, recognize, from an evaluation of the FHR pattern, a compromised fetus before irreversible organ damage occurs, and moderate any deterioration. Failing that, to rescue the fetus from its hostile environment, the key to surveillance is to avoid the need to rescue urgently. The key to resuscitation is to restore adequate oxygenation and perfusion of vital organs, particularly the brain. The Neonatal Resuscitation Program helps individuals learn the cognitive, technical, and teamwork skills to resuscitate and stabilize newborns.

\section{"Almost all neonates born in sub-optimal conditions can be anticipated with careful evaluation of prenatal and intrapartum records. A review of the last hours or minutes of the fetal electronic heart patterns is useful to understand the fetal condition prior to birth."}

Almost all neonates born in sub-optimal conditions can be anticipated with careful evaluation of prenatal and intrapartum records. A review of the last hours or minutes of the fetal electronic heart patterns is useful to understand the fetal condition prior to birth. There should be very few surprises at the time of delivery, but having a skilled team even when the unexpected occurs just before delivery should be managed proficiently. Being prepared is the first and most important step in delivering effective neonatal resuscitation. In the United States, $10 \%$ of all newborns need intervention, $5 \%$ of term newborns receive PPV, $2 \%$ of term newborns are intubated, and $1 \%$ require extensive resuscitative measures. Hospitals should not deliver babies if personnel with the knowledge and skills cannot be immediately available to resuscitate a neonate. Knowledge of the risk factors will help the personnel be prepared well in advance. The following risk factors increase the likelihood of neonatal resuscitation:

- Maternal

diabetes, hypertension, advanced or very young maternal age, multiple gestations, lack of prenatal care, substance abuse

- Fetal

prematurity, post dates, intrauterine growth restriction, macrosomia, multiple gestations, significant fetal anomalies, malformations or anomalies, arrhythmias, hydrops

- $\quad$ Antepartum

oligohydramnios, polyhydramnios, placental or cord abnormalities, premature or prolonged rupture of membranes

- Intrapartum

emergency cesarean delivery, forceps or vacuum-assisted delivery, breech, transverse or other abnormal presentation, category II or III fetal heart rate pattern, excessive uterine activity (tachysystole), maternal general anesthesia, maternal magnesium therapy, placental abruption, intrapartum bleeding, chorioamnionitis, opioids administered 
to mother within 4 hours of delivery, shoulder dystocia, meconium-stained amniotic fluid, prolapsed umbilical cord, maternal boluses containing glucose, precipitous delivery.

"Communication between the obstetrician and the labor and delivery staff with the neonatal resuscitation team is vital. Inquiry of the umbilical cord blood gases values and timely repeated gases after the baby is born is critical in those cases where samples are sent for evaluation. If the hospital does not provide therapeutic hypothermia, then an arrangement with a local facility that does offer the procedure must be in place, with the understanding of the timeliness of early transfer."

Communication between the obstetrician and the labor and delivery staff with the neonatal resuscitation team is vital. Inquiry of the umbilical cord blood gases values and timely repeated gases after the baby is born is critical in those cases where samples are sent for evaluation. If the hospital does not provide therapeutic hypothermia, then an arrangement with a local facility that does offer the procedure must be in place, with the understanding of the timeliness of early transfer. Each resuscitation team member needs to show curiosity and initiative in understanding the fetal history and being prepared for unexpected situations. Thorough knowledge of NRP with frequent drills helps carry out a seamless resuscitation of these vulnerable infants.

\section{Suggested reading:}

American Academy of Pediatrics. Textbook of Neonatal Resuscitation, 8th ed, Weiner GM (Ed), American Academy of Pediatrics, 2021.

Disclosures: The authors have indicated no conflicts of interest.

\section{NT}

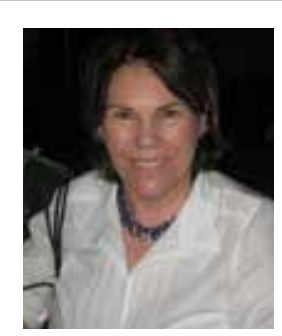

\section{Corresponding Author:}

Maureen E. Sims, M.D.

Professor of Pediatrics

University of California, Los Angeles

Los Angeles, CA

email: mes@g.ucla.edu

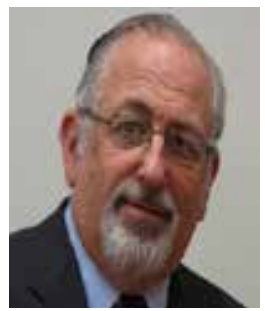

Barry Schifrin, M.D,

Formerly Professor of Obstetrics and Gynecology Western University

Pomona, CA

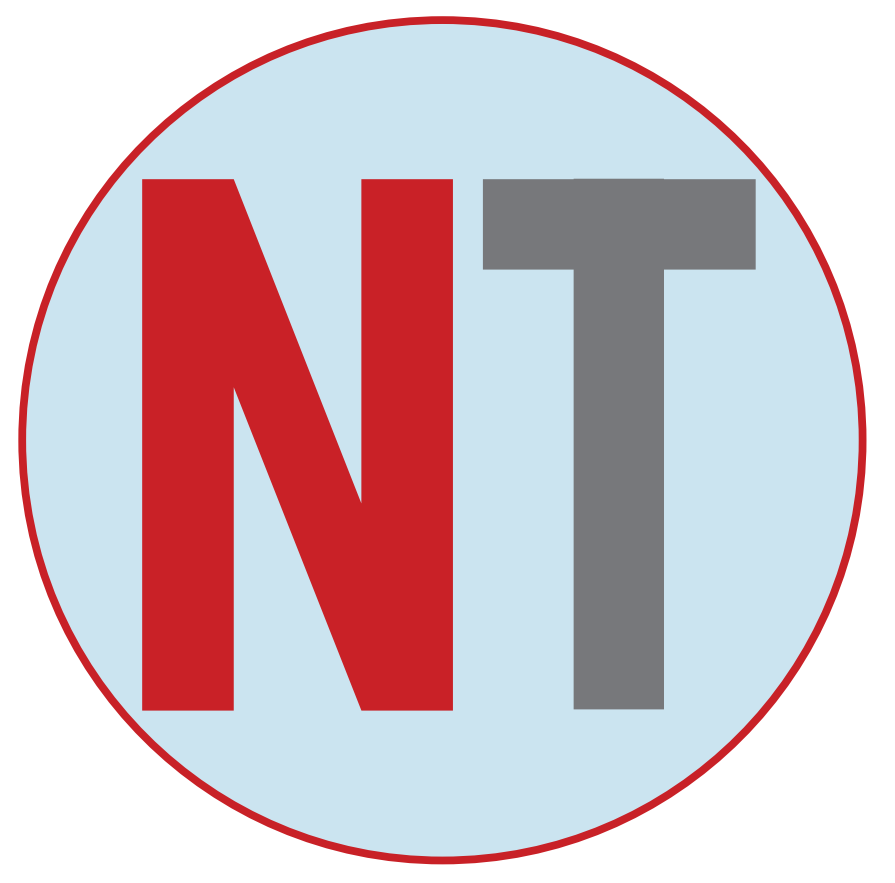

New subscribers are always welcome! NEONATOLOGY IQDAY

To sign up for a free monthly subscription, just click on this box to go directly to our subscription page 